\title{
A model for spin-polarized transport in perovskite manganite bi-crystal grain boundaries
}

\author{
R. Gunnarsson ${ }^{1}$, A. Kadigrobov ${ }^{1,2}$ and Z. Ivanov ${ }^{1}$ \\ ${ }^{1}$ Department of Microelectronics and Nanoscience, Chalmers University of \\ Technology and Göteborg University, S-412 96 Göteborg, Sweden \\ ${ }^{2}$ B. I. Verkin Institute for Low Temperature Physics \& Engineering, National \\ Academy of Science of Ukraine, 47 Lenin Ave., 310164 Kharkov, Ukraine
}

October 29, 2018

\begin{abstract}
We have studied the temperature dependence of low-field magnetoresistance and current-voltage characteristics of a low-angle bi-crystal grain boundary junction in perovskite manganite $\mathrm{La}_{2 / 3} \mathrm{Sr}_{1 / 3} \mathrm{MnO}_{3}$ thin film. By gradually trimming the junction we have been able to reveal the non-linear behavior of the latter. With the use of the relation $M_{G B} \propto M_{b u l k} \sqrt{M R^{*}}$ we have extracted the grain boundary magnetization. Further, we demonstrate that the built-in potential barrier of the grain boundary can be modelled by $V_{b i} \propto M_{b u l k}^{2}-M_{G B}^{2}$. Thus our model connects the magnetoresistance with the potential barrier at the grain boundary region. The results indicate that the band-bending at the grain boundary interface has a magnetic origin.
\end{abstract}

Recent studies have shown that grain boundaries (GBs) in polycrystalline perovskite manganites, so called colossal-magnetoresistance (CMR) materials, give raise to a low-field magnetoresistance. [1, 2] To analyze the contribution from a single grain boundary on magnetoresistance, thin film bi-crystal structures have previously been applied by several research groups. 3, 4, 5, 6, 7, 8, 9, 10, 11

Supported by such studies Evetts et al [12] have suggested that the lowfield magnetoresistance $M R^{*}$ associated with GBs relates to the magnetization $M_{G B}$ of the interface region. In their model they assume that the resistivity $\rho$ depends on the magnetization $M$ as $\rho \propto \rho_{0} \exp \left[-M^{2} / M_{B}^{2}\right]\left(M_{B}\right.$ represents the bulk magnetization) and hence the magnetoresistance $M R \equiv\left(\rho_{0}-\rho\right) / \rho_{0}$ can for the GB be determined as

$$
M R^{*} \propto \frac{M_{G B}^{2}}{M_{B}^{2}}
$$

where $M R^{*}$ is measured at coercive fields and hence $M_{G B}^{2} \ll M_{B}^{2}$.

In addition to the low-field magnetoresistance, non-linear current-voltage (I-V) characteristics has been observed for manganite GBs. [6, 6, 8, 9] It has previously been demonstrated for other perovskite oxides that grain boundaries introduce a band-bending effect. 13, 14] Further Furukawa has demonstrated that for CMR materials the magnetization $M$ induces a shift in the chemical potential with $\Delta \Phi \propto M^{2}$. [15] As a result of the dislocations and crystalline defects, there is a suppressed magnetic order close to the GB, and thus there is a built-in potential barrier associated with the GB region. Hence as discussed by Gross et al [9] this built-in potential barrier originates from the suppressed magnetic order at the paramagnetic GB, due to the difference in chemical potential in the grain boundary region as compared to the bulk. 
These two models, by Evetts et al [12] and by Gross et al [9], describe two different sides of the influence of the grain boundary, the first one on spin-polarized transport and the second on the potential barrier causing the non-linear I-V characteristics. However, until now there is no model that can explain consistently $M R^{*}$ and the band-bending effects observed in perovskite manganites. One obstacle has been the lack of a full set of data for the magnetization in the GB region. Here we present a new approach to the problem. First we argue that the grain boundary magnetization can be found from the low-field magnetoresistance. We then successfully have applied a method to model the appearance of the magnetic potential barrier at the GB. As a result of this study we can show that the origin of the non-linear current-voltage characteristics is a built-in potential barrier with a barrier height that is determined by the difference in square between the bulk and grain boundary magnetizations.

This study has been performed on a symmetric low-angle bi-crystal grain boundary. Previous transmission electron studies have shown that manganite grain boundaries are relatively straight and well defined. [9, 16, 17] As manganite material we have chosen $\mathrm{La}_{2 / 3} \mathrm{Sr}_{1 / 3} \mathrm{MnO}_{3}$ (LSMO) which has the largest oneelectron bandwidth as well as the highest $T_{C}$ among the ordinary perovskite manganites. 18

A LSMO film of the above composition was grown by pulsed laser deposition on a $\mathrm{LaAlO}_{3}$ bi-crystal substrate with symmetric misorientation angle of $8.8^{\circ}$. In this process a stoichiometric target was ablated by an excimer laser $\left(\mathrm{KrF}, \lambda=248 \mathrm{~nm}, \tau=30 \mathrm{~ns}\right.$ ) with an energy density of $\sim 1.4 \mathrm{~J} / \mathrm{cm}^{2}$. During deposition the substrate was held at $740^{\circ} \mathrm{C}$ in oxygen pressure of 0.4 mbar. A high degree of epitaxy of the $90 \mathrm{~nm}$-thick film was verified by X-ray $\theta-2 \theta$ and $\phi$-scans in a four-circle diffractometer. The film was then patterned with photolithography and Ar-ion milling into microbridges crossing the GB and forming grain boundary junctions (GBJs) with a width of $w=6 \mu \mathrm{m}$. The magnetoresistance properties were measured in a helium cryostat with a variable temperature insert $(2 \mathrm{~K}-400 \mathrm{~K})$ and a $5 \mathrm{~T}$ superconducting magnet. In all measurements the magnetic field was in the plane of the film and parallel to the GB, the resistance was measured with a bias current of $10 \mu \mathrm{A}$. The high-field MR has been deduced from the zero-field and field cooled resistance measurements, while the low-field MR was measured at stable temperatures. Current-voltage characteristics at zero external field were obtained by applying dc current and measuring voltage in a four point contact geometry. A single GBJ was trimmed, which revealed the non-linear effect on the I-V curves. The trimming was performed by a focused ion-beam (FIB) with Ga-ion source. The junction was trimmed in two steps, the first FIB-process left a grain boundary junction $2 \mu \mathrm{m}$ wide and $20 \mu \mathrm{m}$ long, while in the second FIB-process the junction was trimmed down to $w=1 \mu \mathrm{m}$. The junction geometry after the last trimming step is shown in Fig. 11.

The Curie temperature $T_{C}$ of the sample before trimming was about $350 \mathrm{~K} .19$ After trimming $T_{C}$ decreased to about $315 \mathrm{~K}$ which could be observed in the resistance and the high-field MR curves (as indicated by the arrows in Fig. (1). The shift may in part be explained by Ga contamination from the FIB-process, as other studies have shown that for manganite materials a $6 \%$ change in Gadoping may cause a decrease of $T_{C}$ with $50 \mathrm{~K}$.20 An other reason could of course be structural disorder mainly at the edges of the junction. However the variation in resistance of the narrower junctions are not more than would be 
expected from geometric considerations. Further trimming of the GBJ (from $2 \mu \mathrm{m}$ to $1 \mu \mathrm{m}$ ) does not change the transition temperature. Moreover the magnitude of the high-field ( $5 \mathrm{~T}$ ) MR is not affected, even though a broadening in the peak can be seen. As the resistance and high-field magnetoresistance primarily are sensitive to the bulk electrode magnetization the $T_{C}$ values can be estimated from that data. Thereby we have found that the Curie temperatures $\left(T_{C}\right)$ are $350 \mathrm{~K}, 315 \mathrm{~K}$ and $315 \mathrm{~K}$ for the $w=6 \mu \mathrm{m}, 2 \mu \mathrm{m}$ and $1 \mu \mathrm{m}$ wide GBJs respective.

In the low-field magnetoresistance we first note that the single GBJ shows a clear multiple-step resistance, similar to what has been presented in previous studies (see e.g. Fig. 2 in Ref. [6]). Next we observe that the peak low-field magnetoresistance occur at a field $H^{*} \approx 30 \mathrm{mT}$ close to the coercive field. We associate the multi-step behavior with domain wall pinning close to the GB. As the junction was trimmed the number of pinning points decreased and the number of steps decreased consistently. For single GBJs the MR pattern was not reproducible, which made us conclude that the domain walls were pinned at different points for every magnetic field sweep. Due to this irreproducibility several scans were made to maximize the $M R^{*}$.

From the temperature dependence of the $M R^{*}$, we can extract the low-field magnetoresistance onset temperature, i.e. the Curie temperature of the grain boundary region, $T_{C}^{*}$. For the $6 \mu \mathrm{m}$ wide GBJ we find $T_{C}^{*} \approx 320 \mathrm{~K}$ and for the narrower GBJs $T_{C}^{*} \approx 280 \mathrm{~K}(2 \mu \mathrm{m})$ and $T_{C}^{*} \approx 260 \mathrm{~K}(1 \mu \mathrm{m})$. Thus, there is a decrease in $T_{C}^{*}$ as the width of the GBJ is decreased. At the same time the ratio $T_{C}^{*} / T_{C}$ decreases slightly from about 0.91 to 0.84 .

The grain boundary magnetization can be found from Eq. (11) as $M_{G B}=$ $M_{B} \sqrt{M R^{*}}$ except for a proportionality constant. The bulk electrode magnetization $M_{B}$ can be obtained from $M_{B} \propto\left(1-T / T_{C}\right)^{\beta}$ where $\beta=0.37$. 212 The general shape of the thereby extracted $M_{G B}$-curves, shown in Fig. 2, closely resembles the surface-boundary magnetization measured by Park et al (Fig. 4 in Ref. [22]). From the estimate of $M_{G B}$ we conclude that the GB region has a certain amount of spontaneous magnetic order in the whole temperature range below $T_{C}^{*}$, where $T_{C}^{*}$ is of the same order as $T_{C}$. Moreover in the vicinity of $T_{C}^{*}$ we can assume that magnetization scales as $m \propto\left(1-T / T_{C}^{*}\right)^{\beta}$ where $\beta$ is the scaling parameter. Such a power-law behavior would lead to a straight line in a plot of $\log \left(M_{G B}\right)$ and $\log \left(1-T / T_{C}^{*}\right)$ as in the inset of Fig. 2. Since the deviation from linear behavior is small in our data we can obtain (from the slope) a rough estimation of the scaling parameter $\beta \approx 1.3$.

As $M_{G B}$ is different from $M_{S}$ we will have different shift in the chemical potential in bulk electrodes and in the GB region as illustrated in Fig. 3. Following the Furukawa suggestion those shifts will be $\Delta \Phi_{b u l k} \propto M_{B}^{2}$ and $\Delta \Phi_{G B} \propto M_{G B}^{2}$. Hence the height of the built-in potential barrier can be written as

$$
V_{b i} \propto \Delta \Phi_{b u l k}-\Delta \Phi_{G B} \propto M_{B}^{2}-M_{G B}^{2}=A^{\prime}\left(M_{B}^{2}-M_{G B}^{2}\right)
$$

where $A^{\prime}$ is a proportionality constant.

This built-in potential acts a tunnel barrier for the charge carriers and therefore more information about $V_{b i}$ can be obtained from the current-voltage characteristics. Several previous studies (see e.g. Refs. [6, 6, 8, 9, 10]) have shown that the Julliere model [23] for tunneling transport between ferromagnetic electrodes is not directly applicable to charge transport across manganite GBs. 
Instead charge transfer across this barrier has recently (see Ref. 91) been described as Glazman-Matveev transport, 24 charge hopping via impurity states in the interface region, which results in an inelastic contribution to the conductivity. The current at low voltages in this study follows $I \propto V^{\alpha}$, where $\alpha=1 \div 2$, which is consistent with previously suggested theories, [6, 8, 9, 10] however due to lack of data points at low-voltages we are not able to distinguish between the models. At larger voltages, about $1 \mathrm{~V}$, the I-V curve is fairly linear up to about $5 \mathrm{~V}$ where Joule heating starts to dominate. From this linear part we have extrapolated a zero bias-current voltage $V_{0}$ as illustrated in Fig. G. (a). Within the error of a pre-factor we assume that $V_{0}$ can give us the temperature dependence of the built-in potential, $V_{b i}$. 25. Thus we have

$$
V_{b i}(T) \propto V_{0}(T)
$$

by the assumption that the proportionality constant is temperature independent.

As shown in Fig. 4(c) the differential conductance changes character at $T_{C}$, however it is still non-linear for temperatures above. This indicates that the non-linear behavior of the I-V curves is not solely due to the difference in magnetization of the GBJ. Above $T_{C}$ the charge carriers travel between the metallic paramagnetic electrodes via the GB layer which forms a low conductivity metalinsulator-metal (M-I-M) junction. Charge transport across the GB may still be Glazman-Matveev type even above $T_{C}$ since their theory does not account for any dependence on magnetic state. The difference in the differential conductance above and below $T_{C}$ indicates that there is a magnetic contribution to the band bending. By the shape and the magnitude of those dependencies it is clear that the major part of the band bending has a magnetic origin and only an insignificant contribution comes from the M-I-M junction. Eq. (2) then models the magnetic contribution to the potential barrier of the GB which is the dominating part below $T_{C}$.

Finally merging Eqs. (2) and (3) the measured temperature dependence of the built-in potential can be obtained from

$$
V_{0}=A^{\prime}\left(M_{B}^{2}-M_{G B}^{2}\right)
$$

where $M_{B} \propto\left(1-T / T_{C}\right)^{0.37}$ and $M_{G B} \propto\left(1-T / T_{C}^{*}\right)^{1.3}$. Now $M_{G B}$ is extracted from our measured data of $M R^{*}$, thus inserting $C$ as the proportionality between $M R^{*}$ and $M_{G B}^{2} / M_{B}^{2}$ in Eq. (11) we will have

$$
V_{0}=A\left(\left(1-T / T_{C}\right)^{2 \cdot 0.37}-1 / C\left(1-T / T_{C}^{*}\right)^{2 \cdot 1.3}\right)
$$

where $A$ is related to $A^{\prime}$ (from Eq. (2)). We then scale this expression (Eq. (5)) with the parameter $A$ to our measured data for $V_{0}$. The result is shown in Fig. 5 for $w=2 \mu \mathrm{m}$ and $w=1 \mu \mathrm{m}$ GBJs $(w=6 \mu \mathrm{m}$ is omitted due to the lack of $V_{0}$ data). Good agreement with the data were found for $C=3$ and $A(2 \mu \mathrm{m})=0.5$ and $A(1 \mu \mathrm{m})=0.39$. We note that the above relation for $M_{B}$ with $\beta=0.37$ is valid only in the vicinity of $T_{C}$ and that the relation for $M_{G B}$ is valid only in the vicinity of $T_{C}^{*}$. However both $M_{B}$ and $M_{G B}$ describe the general behavior of the magnetization curve for bulk and the grain boundary region respective in the whole temperature region. Thus we could use them as a model for magnetization in the entire temperature range below $T_{C}$. From Fig. 5 
we conclude that even though each detail in the $V_{0}$ data is not reproduced by our model it does outline the main behavior of the data.

To summarize we have measured the temperature dependence of the grain boundary magnetization and the magnetic built-in potential barrier of the GB when trimming a LSMO low-angle bi-crystal GBJ. We have thereby employed the proposition of Evetts et al [12 that $M R^{*} \propto M_{G B}^{2} / M_{B}^{2}$. Further we have shown that for low-angle GB the Curie temperature of the GB region $T_{C}^{*}$ is partially suppressed as compared to the bulk $T_{C}$ and the ratio $T_{C}^{*} / T_{C}$ is about 0.9 . Thus the grain boundary magnetization $M_{G B}$ exists close to the bulk Curie temperature [26]. Moreover we have been able to extract the behavior of $M_{G B}$ together with a rough estimate of the scaling parameter $\beta \approx 1.3$.

From the ideas of Gross et al [9] and Furukawa [15] we then have developed a model for the built-in potential barrier for charge carriers. The model is based on the different shift in chemical potential due to difference in magnetization of the GB and the bulk resulting in $V_{b i} \propto M_{B}^{2}-M_{G B}^{2}$. We have then shown that the main features of the temperature dependence of $V_{b i}$ can be resembled with this model. Moreover we have shown that the magnetization of the sample has a much higher impact on the non-linearity of the I-V characteristics than the metal-insulator-metal junction. We attribute this to the high spin polarization of LSMO.27 The observed temperature dependence of $M_{G B}$ and the differential conductance curves has allowed us to draw a schematic phase diagram of the GB (Fig. 4(b)) as a complement to ordinary phase diagrams (see e.g. Ref. 18]). With our model we have been able to connect the low-field magnetoresistance and the non-linear behavior observed in GBJs of perovskite manganite materials.

The work has been supported by The Swedish Research Council (TFR)

and The Board for Strategic Research (SSF) with the programs "OXIDE" and "Transport in mesoscopic structures".

\section{References}

[1] H.Y. Hwang, S.-W. Cheong, N.P. Ong, and B. Batlogg. Phys. Rev. Lett., $77: 2041,1996$.

[2] A. Gupta, G.Q. Gong, Gang Xiao, P.R. Duncombe, P. Lecoeur, P.L. Trouilloud, Y.Y. Wang, V.P. Dravid, and J.Z. Sun. Phys. Rev. B, 54:R15629, 1996.

[3] N.D. Mathur, G. Burnell, S.P. Isaac, T.J. Jackson, B.-S. Teo, J.L. McManus Driscoll, L.F. Cohen, J.E. Evetts, and M.G. Blamire. Nature, $387: 266,1997$.

[4] K. Steenbeck, T. Eick, K. Kirsch, K. O'Donnell, and E. Steinbeiss. Appl. Phys. Lett., 71:968, 1997.

[5] K. Steenbeck, T. Eick, K. Kirsch, H.-G. Schmidt, and E. Steinbeiss. Appl. Phys. Lett., 73:2506, 1998.

[6] N.K. Todd, N.D. Mathur, S.P. Isaac, J.E. Evetts, and M.G. Blamire. J. Appl. Phys., 85:7263, 1999.

[7] W. Westerburg, F. Martin, S. Friedrich, M. Maier, and G. Jakob. J. Appl. Phys., 86:2173, 1999. 
[8] J. Klein, C. Höfener, L. Alff, B. Büchner, and G.M. Gross. Europhys. Lett., $47: 371,1999$.

[9] R. Gross, L. Alff, B. Büchner, B.H. Freitag, C. Höfener, J. Klein, Yafeng Lu, W. Mader, J.B. Philipp, M.S.R. Rao, P. Reutler, S. Ritter, S. Thienhaus, S. Uhlenbruck, and B. Wiedenhorst. J. Magn. Magn. Mater., 211:150, 2000.

[10] C. Höfener, J.B. Philipp, J. Klein, L. Alff, A. Marx, B. Büchner, and R. Gross. Europhys. Lett., 50:681, 2000.

[11] R. Mathieu, P. Svedlindh, R. Gunnarsson, and Z. G. Ivanov. Phys. Rev. B, 63:132407, 2001.

[12] J.E. Evetts, M.G. Blamire, N.D. Mathur, S.P. Isaac, B.-S. Teo, L.F. Cohen, and J. MacManus-Driscoll. Phil. Trans. R. Soc. London A, 356:1593, 1998.

[13] J. Mannhart and H. Hilgenkamp. Supercond. Sci. Technol., 10:880, 1997.

[14] see e.g. the reports from the Electroceramics VI '98, Montreux, Switzerland, 24-27 August 1998, in Journal of the European Ceramic Society, vol. 19 (1999), N. Setter, E.Colla and D. Damjanovic (editors).

[15] N. Furukawa. J. Phys. Soc. Japan, 66:2523, 1997.

[16] A.L. Vasiliev and Z.G. Ivanov. unpublished.

[17] D.J. Miller, Y.K. Lin, V. Vlasko-Vlasov, and U. Welp. J. Appl. Phys., $87: 6758,2000$.

[18] Y. Tokura and Y. Tomioka. J. Magn. Magn. Mater., 200:1, 1999.

[19] The value for $T_{C}$ is based on superconducting interference device (SQUID) magnetometer measurements on other $\mathrm{La}_{2 / 3} \mathrm{Sr}_{1 / 3} \mathrm{MnO}_{3}$ on $\mathrm{LaAlO}_{3}$ bicrystal samples in the same batch.

[20] K. Veera Krishna Meera, V. Ravindranath, and M.S. Ramachandra Rao. J. Alloys and Compounds, 326:98, 2001.

[21] K. Ghosh, C.J. Lobb, R.L. Greene, S.G. Karabashev, D.A. Shulyatev, A.A. Arsenov, and Y. M. Mukovskii. Phys. Rev. Lett., 81:4740, 1998.

[22] J.-H. Park, E. Vescovo, H.-J. Kim, C. Kwon, R. Ramesh, and T. Venkatesan. Phys. Rev. Lett., 81:1953, 1998.

[23] M. Julliere. Phys. Lett., 54A:225, 1975.

[24] L.I. Glazman and K.A. Matveev. Sov. Phys. JETP, 67:1276, 1988.

[25] R. Gunnarsson, A. Kadigrobov, and Z. G. Ivanov. Mat. Res. Soc. Symp. Proc., 674:U3.7, 2001.

[26] We believe that the observed $T_{C}^{*} \ll T_{C}$ from Ref. 9 is because they performed their measurements on bi-crystals with high misorientation-angle.

[27] J.-H. Park, E. Vescovo, H.-J. Kim, C. Kwon, R. Ramesh, and T. Venkatesan. Nature, 392:794, 1998. 


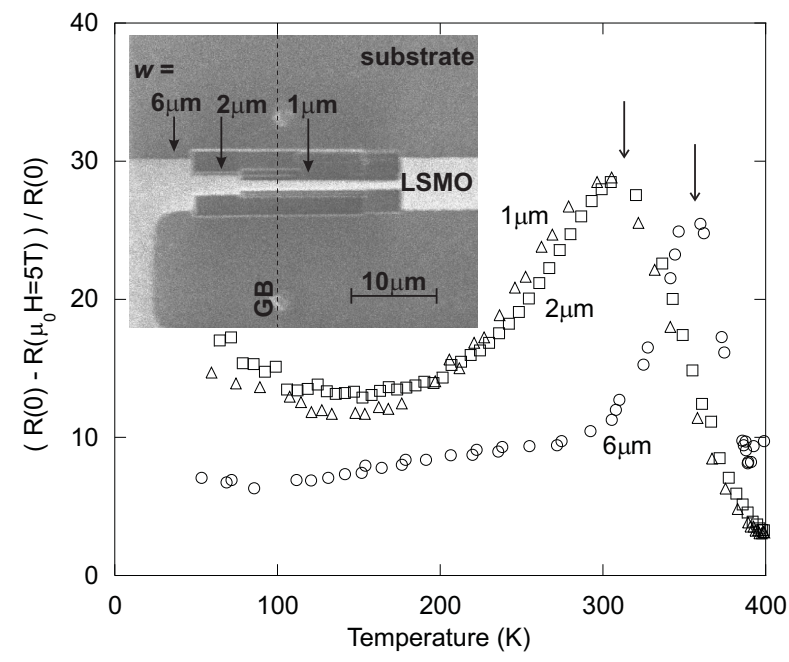

Figure 1: High field (5 T) magnetoresistance as function of temperature. The Curie temperatures $T_{C}$ are indicated with arrows. The inset show a secondaryelectron image of the GBJ geometry after the second trimming process.

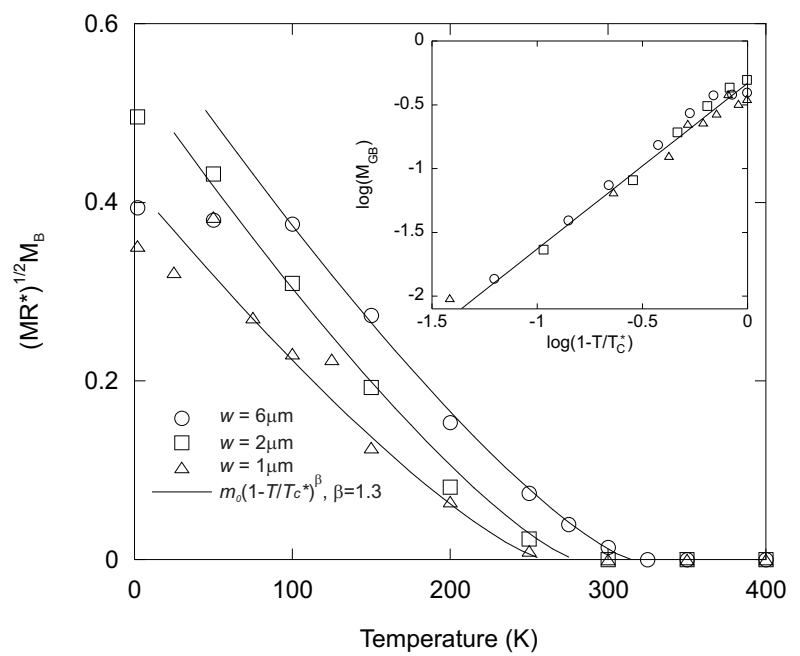

Figure 2: Grain boundary magnetization $M_{G B}$ extracted from $M_{B} \sqrt{M R^{*}}$ and plotted as function of temperature. The lines represent $m_{0}\left(1-T / T_{C}^{*}\right)^{\beta}$ where $\beta=1.3$ is found from the slope of the line in the logarithmic plot (the inset). For $w=6 \mu \mathrm{m}, 2 \mu \mathrm{m}$ and $1 \mu \mathrm{m}, T_{C}^{*}=320 \mathrm{~K}, 280 \mathrm{~K}$ and $260 \mathrm{~K}$ and $m_{0}=0.615$, 0.54 and 0.42 has been used respectively. The data shown correspond to the maximum $M R^{*}$ values obtained. 


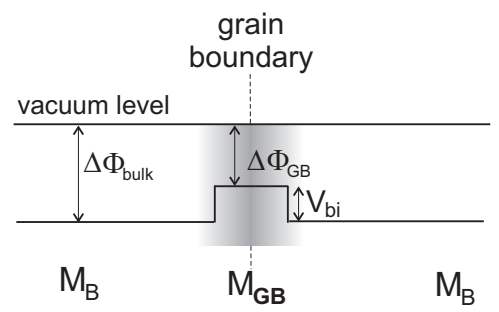

Figure 3: A schematic illustration of the band structure close to the GB. The difference in chemical potential shift due to difference in magnetization of the bulk and the grain boundary region defines the built-in potential $V_{b i}$.

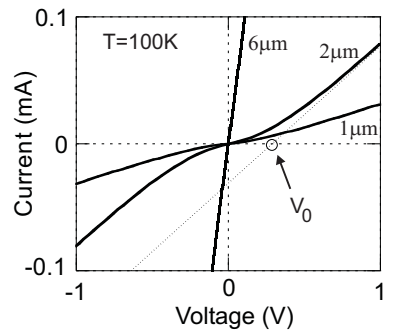

( a )

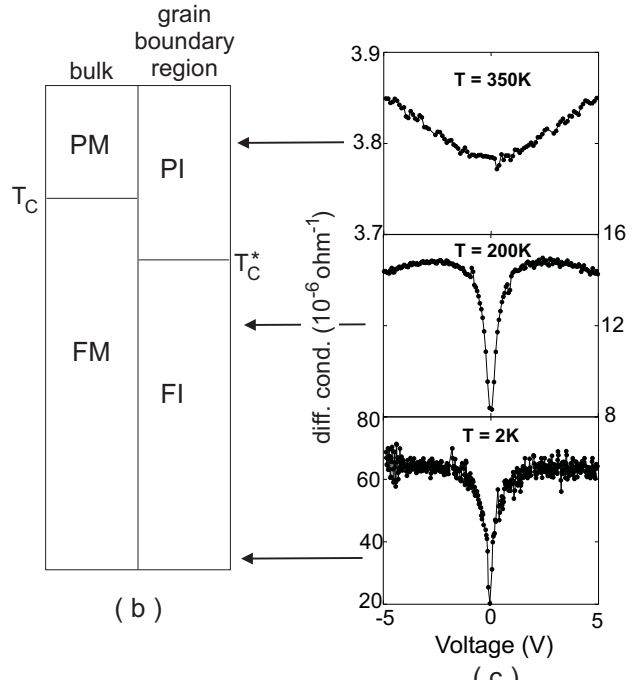

(c)

Figure 4: (a) From the linear part of the non-linear I-V curves we extrapolate a zero bias-current voltage $V_{0}$ as illustrated here for $T=100 \mathrm{~K}$. (b) A scetch of the phase diagram of the GB region given from the observed $M_{G B}$ temperature dependence (see Fig. 2) and the (c) differential conductance curves. The differential conductance is shown for the $w=1 \mu \mathrm{m}$ wide junction at $T=350 \mathrm{~K}$, $200 \mathrm{~K}$ and 2 K. PM, FM, PI and FI represent the paramagnetic metallic, ferromagnetic metallic, paramagnetic insulating and ferromagnetic insulating phases. The transition between PM to FM occur at $T_{C}$ while the PI to FI transition occur at $T_{C}^{*}<T_{C}$. 


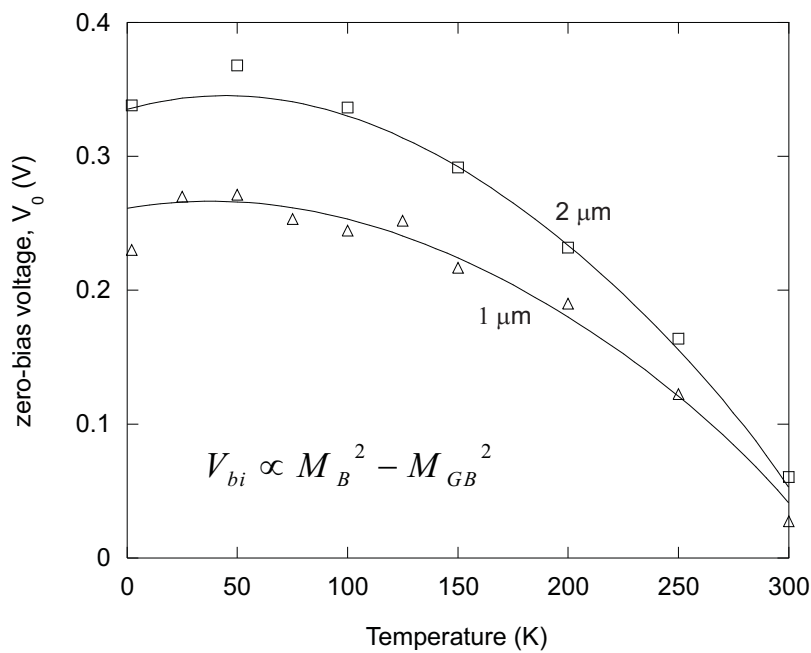

Figure 5: The zero-bias voltage data (open symbols) can be mimiced with our model $V_{0}=A\left(M_{B}^{2}-M_{G B}^{2}\right)$ (lines). Boxes and triangles represent $w=2 \mu \mathrm{m}$ and $1 \mu \mathrm{m}$ respectively. Good agreement with experimental data was found for $A(w=2 \mu \mathrm{m})=0.5$ and $A(w=1 \mu \mathrm{m})=0.39$. 